RAD Conference Proceedings, vol. 2, pp. 207-211, 2017

www.rad-proceedings.org

\title{
MAGNETIC FIELD GRADIENTS AND THEIR EFFECTS ON THE DIFFUSION TENSOR DERIVATE MEASURES
}

\section{Luminita Moraru ${ }^{1 *}$, Lucian Traian Dimitrievici'1,2, Antoaneta Ene ${ }^{1}$, Simona Moldovanu3,4}

${ }^{1}$ Department of Chemistry, Physics and Environment, Faculty of Sciences and Environment, University Dunarea de Jos of Galati, Galati, Romania 2Mihail Kogalniceanu High School, Galati, Romania

3Department of Computer Science and Engineering, Electrical and Electronics Engineering, Faculty of Control Systems, Computers, University Dunarea de Jos of Galati, Galati, Romania 4Dumitru Motoc High School, Galati, Romania

\begin{abstract}
Diffusion tensor imaging (DTI) and the degree of diffusion weighting of the sequence, expressed as the $b$ factor, are used to investigate the effect of the magnetic field gradients on the integrity of white matter in patients with temporal intracerebral hemorrhage. The healthy patients are the gold standard. The present study investigated the changes of mean diffusivity (MD) and fractional anisotropy (FA) in a brain hemisphere approach to understand the effect of magnetic field gradients on the brain hemorrhage investigation. The artifacts induced by diffusion gradients in diffusion tensor imaging affect the accuracy of the investigation, and in order to achieve the optimal image quality, strong magnetic field gradients are recommended. The artifact effect of higher magnetic field gradients is analyzed by means of the root-mean-square FA and MD difference between left and right brain hemispheres.
\end{abstract}

Key words: Magnetic field gradients, diffusion tensor imaging, mean diffusivity, fractional anisotropy, image processing

DOI: $10.21175 /$ RadProc.2017.42

\section{INTRODUCTION}

MRI technique is a noninvasive, three-dimensional, and allows the characterization of the brain. Diffusion tensor imaging (DTI) is a specific application of MRI technique and can evaluate the macroscopic organization in brain tissues.

The diffusion tensor describes both the magnitude of the water diffusion and the directions of anisotropic diffusion. Many studies deal with DTI ability to detect a signal-intensity change in the intracerebral hemorrhage (ICH) case [1-5]. Attempts to quantify and visualize properties of the diffusion tensor have resulted in scalar measures of mean diffusivity (MD) and fractional anisotropy (FA). FA describes the directional dependence of diffusion being related to the axonal fiber density, axonal diameter and myelination in white matter [6]. Thus, FA is very sensitive to microstructural changes. MD measures the mean molecular motion and indicates the complementary aspects of magnetic field-water diffusion-tissue interactions being sensitive to cellularity [7]. However, the identification of the relevant changes in FA and MD is difficult when subjects show high variability in both clinical features and disease severity. Also, intrinsic regional differences between the brain hemispheres lead to misinterpretations [8].

Another source of biased results consists of magnetic field gradients in MRI. MRI has a strong, homogeneous magnetic field Bo that is linearly altered when a gradient pulse is introduced into bore. Usually, MRI scanners are equipped with directional gradient units; a magnetic field gradient can be generated along any arbitrary orientation by combining these units. A first short period of a gradient pulse introduces a phase difference (dephases) between the homogeneous Bo field and gradient directions, depending on the location of the molecules along the gradient axis. The second short period of a gradient pulse rephases the magnetization. In the diffusion measurement, this phase difference is used to detect the water motion. These magnetic field gradients induce artifacts and affect the accuracy of the investigation [9-11]. For an optimal image quality, strong magnetic field gradients are recommended and some investigations may take longer than 20-30 minutes. The diffusion gradients depend on the diffusion weighting expressed as the $\mathrm{b}$-factor $\left(\mathrm{s} / \mathrm{mm}^{2}\right)$. Stronger magnetic gradients mean higher b-factor values.

In order to increase the sensitivity of our analysis, a systematic inter-hemispheres analysis between a

*luminita.moraru@ugal.ro 
healthy brain and ICH alterations in brain diffusion tensor images is performed $[12,13]$.

In this paper we analyze the influence of the choice of b-values on the integrity of white matter in patients with temporal intracerebral hemorrhage $(\mathrm{ICH})$ relative to the healthy patients motivated by the necessity of increasing the magnetic field gradients for better image quality. This study focused on an inter-hemispheres analysis in order to speed up the techniques and as an attempt to avoid a region of interest analysis that is prone to inter and intra- operator sensitive.

\section{MATERIALS AND METHODS}

\subsection{Subjects}

Five subjects with ICH (age range 35 - 69 years; three males and two females) underwent MRI scans. The control group of healthy patients $\mathrm{H}$ (two males and three female) has the mean age 46.66 years \pm 9.03 . The study was approved by the Research Ethics Committee of the Dunarea de Jos University of Galati and Saint Andrew Hospital and a written informed consent was obtained from each participant. Magnetic diffusion gradients were applied in 21 directions and five bvalues were used: b1 $=250 \mathrm{~s} / \mathrm{mm}^{2} ; \mathrm{b} 2=500 \mathrm{~s} / \mathrm{mm}^{2}$; $\mathrm{b} 3=750 \mathrm{~s} / \mathrm{mm}^{2} ; \mathrm{b} 4=1000 \mathrm{~s} / \mathrm{mm}^{2} ; \mathrm{b}_{5}=1250 \mathrm{~s} / \mathrm{mm}^{2}$.

A comparison to the image acquired without diffusion gradients ( $\mathrm{bo}=\mathrm{O} \mathrm{s} / \mathrm{mm}^{2}$ ) is performed.

\subsection{Methods}

The diffusion tensor provides three eigenvalues $\left(\lambda_{1}\right.$, $\lambda_{2}$ and $\lambda_{3}$ ) that characterize the magnitude of the diffusion system and the three associated eigenvectors for the direction of the diffusion system. Mean diffusivity (MD) is the average of the three eigenvalues:

$$
M D=\frac{\lambda_{1}+\lambda_{2}+\lambda_{3}}{3}
$$

The fractional anisotropy (FA) can range from o to 1. o indicates completely random diffusion (isotropic diffusion) and 1 represents total directional diffusion (anisotropic diffusion). It is used as a composite index of water diffusivity changes in each voxel at any directions after the tissue damage:

$$
F A=\sqrt[3]{\frac{3\left[\left(\lambda_{1}-\lambda_{2}\right)^{2}+\left(\lambda_{1}-\lambda_{3}\right)^{2}+\left(\lambda_{3}-\lambda_{2}\right)^{2}\right]}{2\left(\lambda_{1}^{2}+\lambda_{2}^{2}+\lambda_{3}^{2}\right)}}
$$

Many of brain injuries lead to the degeneration of the barriers to free diffusion of hydrogen in white matter degenerate. As a result, MD increases and FA decreases as the diffusion becomes more isotropic.

This study aims to find the effect of strong magnetic gradient fields in FA $(\triangle F A)$ and $\mathrm{MD}(\triangle M D)$ values and their bias $\left(\Delta F A_{\text {left;right }}\right)$ and $\left(\Delta M D_{\text {left;right }}\right)$ between the affected and reference data using a hemisphere approach:

$$
\Delta F A_{\text {left }}=\sqrt{\sum_{i}\left(F A_{\text {left }}^{I C H}-F A_{\text {left }}^{H}\right)^{2}}
$$

and

$$
\Delta F A_{\text {right }}=\sqrt{\sum_{i}\left(F A_{\text {right }}^{I C H}-F A_{\text {right }}^{H}\right)^{2}}
$$

where $i \in\{$ number of subjects $\}$. Similar relationships are used for MD.

For all b-value and hemisphere combinations a paired t-test has been performed and its results indicated that FA and MD are significant for the analyzed brain tissues with 95\% confidence. For a quick visualization of the tissue differences, the histographic characteristics of the brain hemispheres in DTI images for various magnetic gradient fields were used.

\subsection{Image processing}

For the data acquisition, a $1.5 \mathrm{~T}$ MRI scanner (Philips Medical Systems, Best, the Netherlands) with a six-channel sensitivity encoding (SENSE) for faster scanning $(\mathrm{FS}=1.5)$ was used. The scanning parameters were: $3 \mathrm{D}$ gradient echo, the echo time from 83 to $110 \mathrm{~ms}$, and the repetition time varied between the subjects in the range between $6500 \mathrm{~ms}$ and $7800 \mathrm{~ms}$; bandwidth $=1070 \mathrm{~Hz} /$ pixel; flip angles $=2-$ and 6-; voxel resolution $=2.5-3.0 \mathrm{~mm}$; slice thickness $=$ $4 \mathrm{~mm}$, and 128 slices. A DICOM (Digital Imaging and Communications in Medicine) dataset with a matrix size of 116x $92 \times 42$ and a spatial resolution of $2 \times 2 \times 3.6 \mathrm{~mm}$ is used.

To remove residual anatomical differences, an in-house software written in MATLAB for the brain tissues segmentation for skull and background was used $[14,15]$. Each DTI datasets were aligned using the midline inter-hemispheric fissure to compensate for individual differences in the brain. The sagittal axis has been detected using the Hough transforms and each image was split into the left and right hemispheres [16].

\section{RESULTS}

No statistically significant differences were found in the MD and FA values characteristic for left and right hemispheres. In this very primarily step, the absence of statistical differences between DTI-measures from all analyzed b-values can be viewed as an equal diagnostic performance. Further studies dealing with the histograms distribution and on biased values were required.

Figure 1 shows an example of the intensity signal distribution for various b-values in a hemisphere approach. The natural differences between left and right hemispheres are highlighted. Also, the effect of the increasing magnetic gradient fields can be observed, in a comparative approach, between bo $=0$ $\mathrm{s} / \mathrm{mm}^{2}$ that corresponds to the static magnetic field and various b-values corresponding to magnetic field gradients. The pixels distribution changes from a median distribution of the gray scale $[0,255]$ to the white pixels range. The effect of the strong magnetic field is visible for $b=750,1000,1250 \mathrm{~s} / \mathrm{mm}^{2}$ when a shift of the center of the pixels distribution to the median distribution of the gray scale $[0,255]$ appears. 
Also, for higher magnetic field gradients histograms are slightly narrow.

Healthy subject
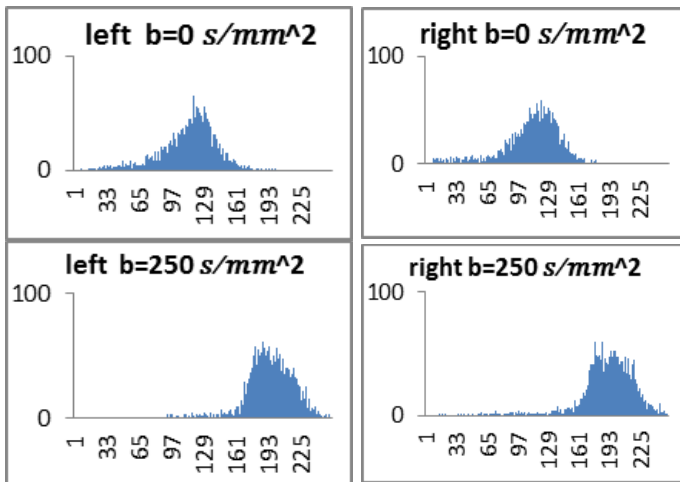

100
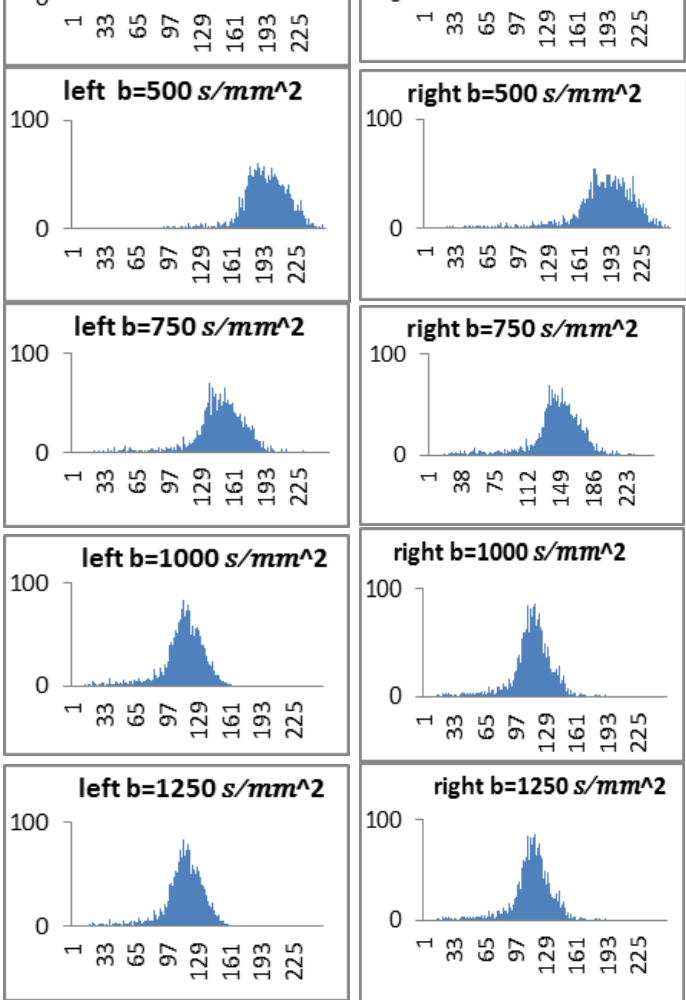

ICH subject
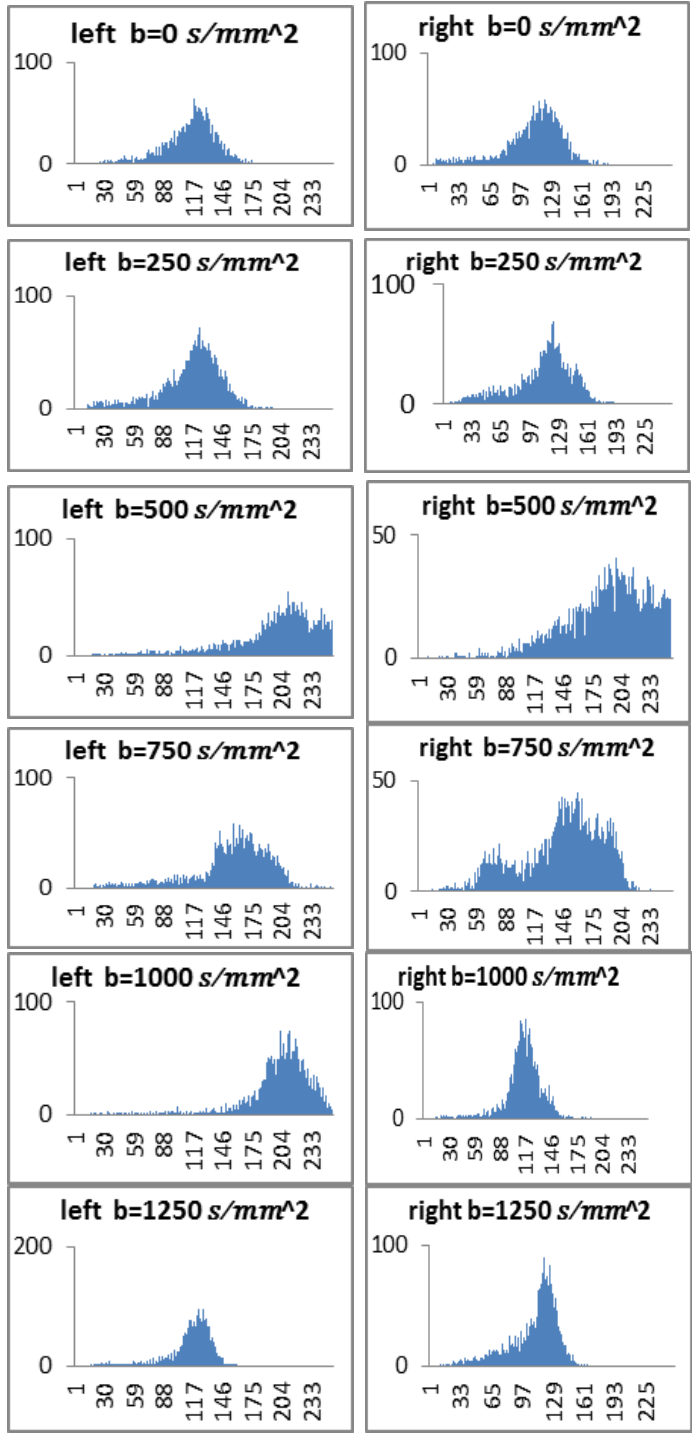

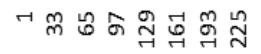

Figure 1. Examples of histograms of the left and right brain hemispheres for a healthy and ICH subject for different magnetic field gradients 
The quantification of the bias in FA and MD for ICH and healthy subjects using the root-mean-square FA and MD difference between the affected and reference data within a brain hemisphere approach is presented in Figure 2.

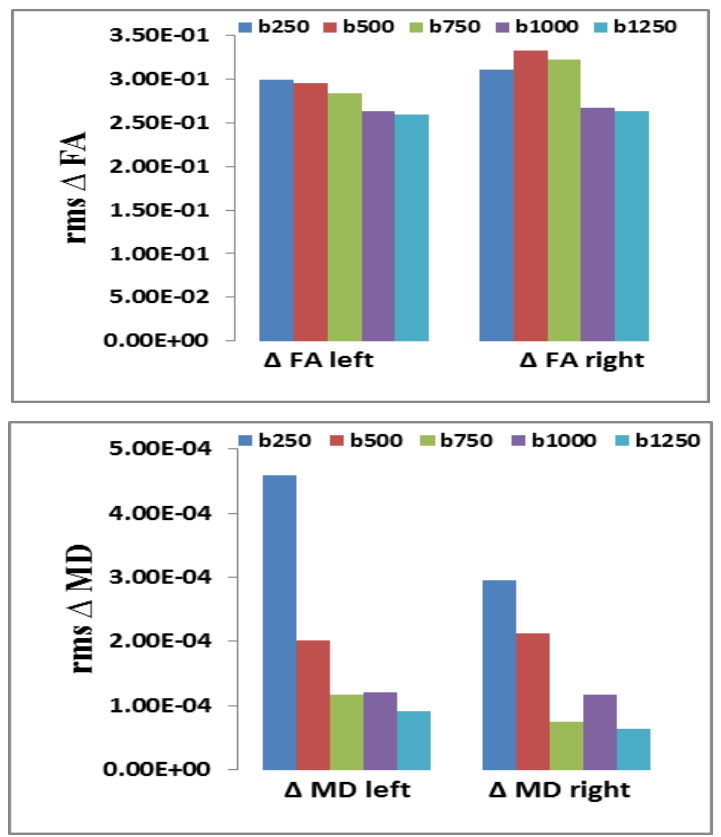

Figure 2. The bias in FA and MD by using the root-meansquare difference between the subjects affected by ICH compared with healthy subjects, for various magnetic field gradients

For lower b-value combinations, $(\triangle F A)$ and $\mathrm{MD}$ $(\triangle M D)$ were significantly different between the left and right hemisphere, whereas for higher b-values these differences are minimized.

\section{DisCUSSION}

In this study, we have shown the effect of magnetic field gradients on the pixel distribution in diffusion tensor images and on diffusivity measures, such as FA and MD. Two DTI data sets were acquired; one belongs to healthy patients and another to the subjects with ICH. The results suggested, in the hemorrhage cases, the average FA values decreased. Our data are in agreement with the plethora of research studies that reported the reduction of $\mathrm{FA}$ in a case of various brain diseases. Also, MD increases and this could be correlated to the increase of the tissue water content after intracerebral hemorrhage.

In the high $b$-value $\left(b=1000,1250 \mathrm{~s} / \mathrm{mm}^{2}\right)$ DTIs, the image quality is visually improved because the diffusion gradient attenuated the signal intensity variation. Comparison with the reference data of healthy patients showed that higher gradients efficiently reduced bias in both FA and MD by reducing the artifacts.

The hemisphere-brain histograms of DTI are sensitive to b-value. At higher b-values a shift of the center of the pixels distribution to the median distribution of the gray scale $[0,255]$ has been observed. Also, for higher magnetic field gradients histograms are slightly narrow. In addition, a comparison analysis of the FA and MD diffusivity measures between the right and left brain hemispheres of the affected and reference data indicates that strong diffusion gradients improve the acquisition process and minimize the variability of quantitative DTI measures.

The common artifacts that affect the DTI are induced by eddy currents, mechanical vibration, susceptibility differences or ghost-like artifacts. They should be minimized in order to support better image quality. One way to avoid and correct them is to increase the magnetic field gradients.

\section{CONCLUSION}

We have presented that, in the presence of strong magnetic field gradients, the effect of artifacts induced by diffusion gradients in diffusion tensor imaging is reduced and the image quality is improved. Quantification of the bias in FA and MD diffusivity measures for using the root-mean-square difference between ICH affected and reference (healthy subjects) data showed that strong magnetic field gradients efficiently reduced bias in FA and MD in a hemispheric approach.

\section{REFERENCES}

1. P. Mukherjee, "Diffusion tensor imaging and fiber tractography in acute stroke," Neuroimaging Clin. $N$. Am., vol. 15, no. 3, pp. 655 - 665, Aug. 2005.

DOI: $10.1016 /$ j.nic.2005.08.010 PMid: 16360595

2. $\quad$ F. Buffon et al., "Longitudinal diffusion changes in cerebral hemispheres after MCA infarcts," J. Cereb. Blood Flow Metab., vol. 25, no. 5, pp. 641 - 650, May 2005. DOI: $10.1038 /$ sj.jcbfm.9600054 PMid: 15689956

3. Q. Yang et al., "Serial study of apparent diffusion coefficient and anisotropy in patients with acute stroke," Stroke, vol. 30, no. 11, pp. 2382 - 2390, Nov. 1999.

DOI: 10.1161/01.STR.30.11.2382 PMid: 10548675

4. F. Zelaya et al., "An evaluation of the time dependence of the anisotropy of the water diffusion tensor in acute human ischemia," Magn. Reson. Imaging, vol. 17, no. 3, pp. 331 - 348, Apr. 1999. DOI: 10.1016/So730-725X(98)oo192-1

5. $\quad$ C. T. Hsieh et al., "Role of diffusion tensor imaging in a patient with spontaneous intracerebral hematoma treated by stereotactic evacuation," Surg. Neurol., vol. 70, no. 1, pp. 75 - 78, Jul. 2008.

DOI: 10.1016/j.surneu.2007.04.004 PMid: 17707485

6. S. Mori, "Mathematics of diffusion measurement," in Introduction to Diffusion Tensor Imaging, Amsterdam, The Netherlands: Elsevier Science \& Technology, 2007, ch. 3, pp. $19-23$. DOI: $10.1016 /$ B978-044452828-5/50017-X

7. A. L. Alexander et al., "Characterization of cerebral white matter properties using quantitative magnetic resonance imaging stains," Brain Connectivity, vol. 1, no. 6, pp. 423-446, Apr. 2012.

DOI: 10.1089/brain.2011.0071

PMid: 22432902

PMCid: PMC3360545 
8. S. Moldovanu et al., "Edge-based structural similarity analysis in brain MR Images," JMIHI, vol. 6, no. 2, pp. 539 - 546, Apr. 2016.

DOI: 10.1166/jmihi.2016.1691

9. D. Gallichan et al., "Addressing a systematic vibration artifact in diffusion-weighted MRI," Hum. Brain Mapp., vol. 31, no. 2, pp. 193 - 202, Feb. 2010. DOI: $10.1002 / \mathrm{hbm} .20856$

PMid: 19603408

10. S. Mohammadi, H. E. Möller, H. Kugel, D. K. Müller, M. Deppe, "Correcting eddy current and motion effects by affine whole-brain registrations: evaluation of three-dimensional distrortions and comparison with slicewise correction," Mag. Res. Med., vol. 64, pp. 1047 - 1056, 2010.

DOI: $10.1002 / \mathrm{mrm} .22501$

PMid: 20574966

11. S. Mohammadi, et al., "Correction of Vibration Artifacts in DTI Using Phase-Encoding Reversal," Magn. Reson. Med., vol. 68, no. 3, pp. $882-889$, Sep. 2012.

DOI: $10.1002 / \mathrm{mrm} .23308$

PMid: 22213396

PMCid: PMC 3569871

12. L. Moraru, S. Moldovanu, A. Biswas, "Intensity based classification and related methods in brain MR images," in Classification and Clustering in Biomedical Signal Processing, N. Dey, A. Ashour,
Eds., Hershey (PA), USA: IGI Global, 2016, ch. 4 pp. $78-105$

DOI: 10.4018/978-1-5225-0140-4.choo4

13. L. Moraru, S. Moldovanu, D. Bibicu, M. Stratulat, "Hemorrhage detection in MRI brain images using images features," in AIP Conf. Proc., Timisoara, Romania, 2013, pp. $171-177$.

DOI: $10.1063 / 1.4832814$

14. S. Moldovanu et al., "Robust Skull Stripping Segmentation based on Irrational Mask for Magnetic Resonance Brain Images," J. Digit. Imaging, vol. 28 , no. 6, pp. $738-747$, Mar. 2015.

DOI: $10.1007 / \mathrm{s} 10278-015-9776-6$

PMid: 25733013

PMCid: PMC4636724

15. M. V. Punga et al., "Level set method coupled with Energy Image features for brain MR image segmentation," Biomed. Tech., vol. 59, no. 3, pp. 219 -229 , Jun. 2014

DOI: 10.1515/bmt-2013-0111

PMid: 24598830

16. S. Moldovanu et al., "The Hough transform and the FSIM similarity index for the sagittal axis identification," Annals of "Dunarea de Jos" University of Galati Mathematics, Physics, Theoretical Mechanics, vol. 38, no. 1, pp. $5-14$, 2015. 